\title{
Filamin C promotes Iymphatic invasion and lymphatic metastasis and increases cell motility by regulating Rho GTPase in esophageal squamous cell carcinoma
}

\author{
Kan Tanabe ${ }^{1,2, *}$, Yoshinari Shinsato ${ }^{2, *}$, Tatsuhiko Furukawa ${ }^{2,4}$, Yoshiaki Kita ${ }^{1}$, \\ Kazuhito Hatanaka ${ }^{3}$, Kentaro Minami², Kohichi Kawahara², Masatatsu Yamamoto ${ }^{2}$, \\ Kenji Baba ${ }^{1}$, Shinichiro Mori ${ }^{1}$, Yasuto Uchikado ${ }^{1}$, Kosei Maemura ${ }^{1}$, Akihide \\ Tanimoto $^{3}$, Shoji Natsugoe ${ }^{1}$ \\ ${ }^{1}$ Department of Digestive Surgery, Breast and Thyroid Surgery, Graduate School of Medical and Dental Sciences, Kagoshima \\ University, Kagoshima, Japan \\ ${ }^{2}$ Department of Molecular Oncology, Graduate School of Medical and Dental Sciences, Kagoshima University, Kagoshima, Japan \\ ${ }^{3}$ Department of Molecular and Cellular Pathology, Graduate School of Medical and Dental Sciences, Kagoshima University, \\ Kagoshima, Japan \\ ${ }^{4}$ Center for the Research of Advanced Diagnosis and Therapy of Cancer, Graduate School of Medical and Dental Sciences, \\ Kagoshima University, Kagoshima, Japan \\ ${ }^{*}$ These authors contributed equally to this work
}

Correspondence to: Tatsuhiko Furukawa, email: furukawa@m3.kufm.kagoshima-u.ac.jp Keywords: FLNC, ESCC, Rac1, Cdc42, migration

Received: September 08, $2016 \quad$ Accepted: December 13, 2016

Published: December 22, 2016

\section{ABSTRACT}

To establish treatments to improve the prognosis of cancer patients, it is necessary to find new targets to control metastasis. We found that expression of FilaminC (FLNC), a member of the actin binding and cross-linking filamin protein family is correlated with lymphatic invasion and lymphatic metastasis in esophageal squamous cell carcinoma (ESCC) by increasing cell motility through activation of Rho GTPase.

Immunohistochemistry analysis showed that FLNC expression in ESCC is associated with lymphatic invasion, metastasis, and prognosis. FLNC knockdown in esophageal cancer cell lines decreased cell migration in wound healing and transwell migration assays, and invasion in transwell migration assays. Furthermore, FLNC knockdown reduced the amount of activated Rac-1 (GTP-Rac1) and activated Cdc42 (GTP-Cdc42). Our results suggest that FLNC expression is a useful biomarker of ESCC metastatic tendency and that inhibiting FLNC function may be useful to control the metastasis of ESCC.

\section{INTRODUCTION}

Invasion and metastasis are fundamental characters of cancer $[1,2]$. The TNM classification of the International Union Against Cancer lists the major prognostic factors in cancer as tumor volume, depth of invasion, and presence of metastasis in lymph nodes or in distant organs [3]. Thus, cancer therapies focus on cancer cell invasion and mechanism of metastasis. However, the ability of cancer cells to migrate is an important element of cancer invasion and metastasis.

Cell migration is an actin-dependent process, involving the polymerization, de-polymerization, branching, and crosslinking of actin filaments [4]. Filamins are a family of actin binding and cross-linking proteins, originally extracted from chicken gizzard muscle [5]. Three highly conserved filamin isoforms have been identified in human: filamin A (FLNA;
ENSG00000196924), filamin B (FLNB; ENSG00000136068), and filamin C (FLNC; ENSG00000128591) [6]. FLNA and FLNB are widely expressed in human tissues while FLNC is restrictively expressed in skeletal and cardiac muscle [7]. All three filamins cross-link actin filaments into three-dimensional structures, and link them to cellular membranes allowing them to serve as scaffolds for transmembrane receptors, channels, signaling molecules, and transcription factors [8,9].

Several studies have described that FLNA and FLNB interact with a number of proteins to regulate signaling events involved in cell shape and migration in cancer [10-17]. In nasopharyngeal and gastric cancer, FLNA expression is correlated with poor prognoses $[15,16]$.

However, there are fewer reports associating FLNC with cancer, and the results are inconsistent [18-21]. To understand the oncological role of FLNC, we studied 
esophageal squamous cell carcinoma (ESCC). ESCC is the most common esophageal cancer in Asian countries [22], and is associated with poor prognosis due to the tendency of local invasion and metastasis [23, 24]. Therefore, local invasion and metastasis is one of the greatest obstacles of successful ESCC treatment.

Here, we performed immunohistochemistry (IHC) to evaluate FLNC protein expression in ESCC tissues and its relationship with clinicopathological factors. We then examined the function of FLNC in ESCC cells using short hairpin RNA (shRNA) interference. Given that Rho GTPase plays an important role in cell motility and the actin filament system, we examined the role of Rho GTPase in ESCC to elucidate how FLNC promotes cell motility. Our data indicate that FLNC expression is associated with lymphatic invasion and lymphatic metastasis and prognosis. Furthermore, we show that FLNC expression increases cell migration and invasion by regulating Rho GTPase.

\section{RESULTS}

\section{Relationship between FLNC expression and clinicopathological findings}

To determine whether the expression of FLNC protein is associated with ESCC patient prognosis, we examined 75 cases of advanced ESCC without superficial carcinoma (Table 1). All patients had received radical surgery without neoadjuvant chemotherapy or radiotherapy. IHC analysis showed that FLNC is predominantly expressed in the cytoplasm of ESCC cells (Figure 1). The biological information of interactions between cancer and the extracellular matrix (ECM) is reflected by histological characteristics at the invasive front, rather than the body of the tumors [25]. Therefore, we evaluated FLNC expression in a marginal portion, within $2 \mathrm{~mm}$ of the invasive external edge of the tumors as described previously [26]. Patients were divided into two groups based on whether levels of FLNC expression were lower or higher than the average. Expression levels were measured as ratio of all patients and the percentages of the low and high FLNC expression groups were $30.7 \%$ (23/75 patients) and 69.3\% (52/75 patients), respectively. FLNC expression was associated with lymphatic metastasis $(\mathrm{N})(P=0.032)$ and lymphatic invasion (ly) $(P=0.032)$. However, there were no significant associations between FLNC expression and age, gender, histological grade, depth of invasion (T), venous invasion (v), or pathological stage (pStage) (Table 1). However, FLNC expression and pStage trend to correlate $(p=0.051)$.

\section{Relationship between FLNC expression and prognosis}

Disease free survival was not significantly different between patients with high and with low FLNC expression
(Supplementary Figure S1). However, overall survival of patients in the high FLNC expression group was significantly shorter than the survival of patients in the low FLNC expression group $(P=0.0135)$ (Figure 2$)$. These data indicate a correlation between high FLNC expression and poor prognosis in patients with ESCC.

\section{Univariate and multivariate analyses of survival}

Univariate analysis showed that the significant prognostic variables for postoperative survival were ly $(P=0.009)$, pStage $(P=0.002)$, and FLNC expression $(P=0.047)$ (Table 2$)$. Multivariate analysis using the above five parameters indicated that only ly $(P=0.018)$ was an independent prognostic factor. FLNC expression was not an independent prognostic factor.

\section{Generation of FLNC knock-down cells}

To examine the functions of FLNC, we first established FLNC knockdown ESCC cell lines from TE-1 and TE- 8 cells using lentiviral delivery of shRNA. To determine the specific effect of FLNC knockdown, we selected FLNC shRNAs that do not affect the expression of FLNA and FLNB in each cell line. TE-1 cells were infected with lentivirus containing either Scrambled shRNA (SshRNA) or FLNC shRNA (FLNCsh1 and FLNCsh3), TE-8 cells were infected with lentivirus containing either SshRNA or FLNC shRNA2 and FINCshRNA3. After selection of infected cells, GFP positive cells accounted for more than $90 \%$ of cells per microscope field $(\times 100)$, for each shRNA (Figure 3A). We evaluated the expression of FLNA, FLNB, and FLNC using real-time PCR and immunoblotting (Figure 3B and 3C). FLNA and FLNB expression levels in FLNC shRNA infected cells were almost equivalent to those in SshRNA infected cells, indicating that FLNC shRNA specifically inhibited FLNC expression.

\section{Increased ESCC cell migration and invasion upon FLNC expression}

We compared cells with FLNC shRNA mediated FLNC knockdown with SshRNA infected cells as control cells. The MTT assay showed that the proliferative abilities of TE-1 and TE-8 FLNC knock-down cells were almost same as those of the control cells (Supplementary Figure S2). Wound healing assays show that the width of the wounds in FLNC knock-down cells was much wider than in the controls after 12 or 6 hours (Figure 4A and 4B). Additionally, FLNC knockdown decreased the number of migratory and invasive cells that passed through the chamber in transwell migration and invasion assays (Figure 4C) Moreover, FLNC knockdown decreased invasion/migration rate. These data indicate that FLNC knockdown decreased the migratory and invasive abilities of cancer cells (Figure 4D). 
Table 1: Relationship between FLNC expression in ESCC and clinicopathologic findings

\begin{tabular}{|c|c|c|c|c|c|c|c|}
\hline & & & & $\mathrm{NC}$ ex & ression & & \\
\hline & Total $n=75$ & $\%$ & High $n=52(69.3 \%)$ & $\%$ & low $n=23(30.7 \%)$ & $\%$ & $P$-value \\
\hline Age $($ mean \pm SD) & $64.9 \pm 8.99$ & & $65.3 \pm 8.53$ & & $64.1 \pm 10.11$ & & NS \\
\hline Gender & & & & & & & 0.650 \\
\hline Male & 61 & 81.3 & 43 & 82.7 & 18 & 78.3 & \\
\hline Female & 14 & 18.7 & 9 & 17.3 & 5 & 21.7 & \\
\hline Histlogy & & & & & & & 0.268 \\
\hline Well & 32 & 42.7 & 20 & 38.5 & 12 & 52.2 & \\
\hline Moderate/Poor & 43 & 57.3 & 32 & 61.5 & 11 & 47.8 & \\
\hline pT & & & & & & & 0.702 \\
\hline pT2 & 11 & 14.7 & 8 & 15.4 & 3 & 13.0 & \\
\hline $\mathrm{pT} 3 / \mathrm{T} 4$ & 64 & 85.3 & 44 & 84.6 & 20 & 87.0 & \\
\hline $\mathrm{pN}$ & & & & & & & 0.032 \\
\hline $\mathrm{pN} 0$ & 23 & 30.7 & 12 & 23.1 & 11 & 47.8 & \\
\hline $\mathrm{pN} 1 / \mathrm{N} 2 / \mathrm{N} 3$ & 52 & 69.3 & 40 & 76.9 & 12 & 52.2 & \\
\hline Lymphatic invasion & & & & & & & 0.032 \\
\hline Negative & 23 & 30.7 & 12 & 23.1 & 11 & 47.8 & \\
\hline Positive & 52 & 69.3 & 40 & 76.9 & 12 & 52.2 & \\
\hline Venous invasion & & & & & & & 0.238 \\
\hline Negative & 8 & 10.7 & 7 & 13.5 & 1 & 4.3 & \\
\hline Positive & 67 & 89.3 & 45 & 86.5 & 22 & 95.7 & \\
\hline pStage & & & & & & & 0.051 \\
\hline II & 24 & 32.0 & 13 & 25.0 & 11 & 47.8 & \\
\hline III/IV & 51 & 68.0 & 39 & 75.0 & 12 & 52.2 & \\
\hline
\end{tabular}

NS: not significant.

Statistical analyses of two group differences were performed using the $\chi^{2}$-test. $P<0.05$ was considered statistically significant.

\section{Decrease of activated Rho GTPase following FLNC knockdown}

Rho family members play important roles in regulating cytoskeletal dynamics. Therefore, we hypothesized that FLNC decreses Rho-mediated cell motility. To investigate how FLNC knockdown inhibits ESCC cell motility, we examined Rho GTPase activation (Figure 5). The levels of activated Rac1 (ENSG00000136238) (GTP-Rac1) and Cdc42 (ENSG00000070831) (GTP-Cdc42) were clearly reduced in FLNC knockdown cells compared with SshRNA infected cells. We could not detect GTP-RhoA (ENSG00000067560) in either TE-1 or TE-8 cells.

\section{DISCUSSION}

Numerous studies have reported that expression of FLNA and FLNB promote cancer invasion and metastasis [12-17]. Meanwhile, the role of FLNC in cancer remains controversial [18-21]. FLNC expression in glioma has been reported to increase with advancing tumor grade, and serum anti-FLNC autoantibody can be a potential biomarker for early glioma diagnosis [19]. However, the expression of FLNC in prostate and gastric cancer was lower than in normal tissues and overexpression of FLNC reduced the invasive abilities of these cell lines [21]. Moreover, in prostate, leukemia, and breast cancer, high expression of FLNC mRNA has been associated with better prognosis by 'in silico' analysis [21]. The conclusions of these reports are inconsistent and the role of FLNC in cancer remains unclear.

Biological information about cancer and interactions between cancer and the ECM is reflected by histological characteristics at the invasive front of the lesion rather than in the body of the tumor [25]. Therefore, we assessed FLNC expression in marginal portion of the tumors, within $2 \mathrm{~mm}$ of the invasive external edge of the tumor [26]. We observed that FLNC expression was significantly associated with lymphatic metastasis, lymphatic invasion, and clinical stage. Moreover, ESCC patients with high FLNC expression had poorer prognosis, consistent with the fact that lymphatic metastasis is a serious prognostic factor for ESCC [23]. We propose that FLNC expression affects the prognosis of ESCC patients by promoting lymphatic invasion and lymphatic metastasis. Our clinical data is similar to results observed for FLNC expression in glioma tissues, but differs from those observed in prostate cancer, breast cancer, and leukemia [19-21]. 
We then examined the role of FLNC in ESCC cell lines with have high levels of FLNC expression. We knocked-down FLNC expression in ESCC cells using shRNA. Wound healing assays and transwell migration and invasion assays showed that FLNC promoted cell migration and invasion in ESCC. Interestingly, the opposite has been reported for gastric and prostate cancer cells, where FLNC expression inhibited cell migration and invasion [21]. We consider that the role of FLNC may differ depending tissue and/or tumor type.

Our results indicate that the function of FLNC resembles that of FLNA. FLNA promotes cancer cell migration and invasion $[11,12,27]$, and regulates actin remodeling by activating Rho GTPase [28, 29].
Rho GTPase plays an important role in regulating the organization of the actin filament system [30-33]. GTPase family members have two conformations, an active GTP-bound conformation, and an inactive GDPbound conformation. Rho GTPase activation assays showed that FLNC knockdown reduced GTP-Rac1 and GTP-Cdc42 levels. Rac1 is involved in the formation of membrane ruffles and the protrusions at the leading edge of migrating cells, lamelipodia [34]. Cdc42 is involved in the formation of filopodia, which function to probe the surrounding environment and control the direction of cell migration [32, 34, 35]. RhoA also leads to cell motility by maintaining focal adhesions and in the assembly of stress fibers [32, 34]. However, GTP-RhoA expression
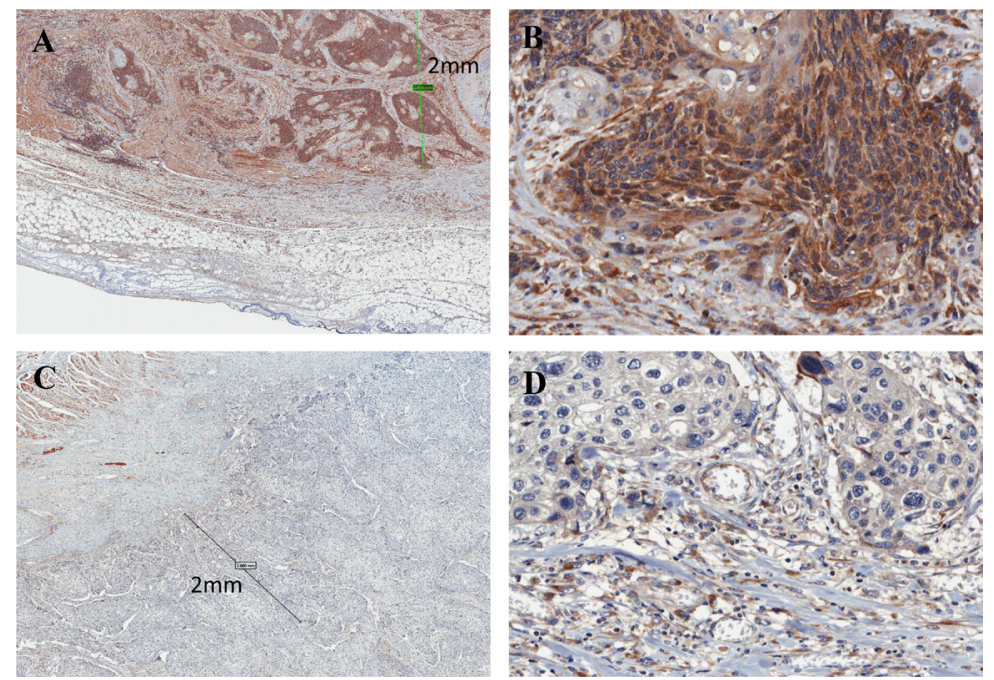

Figure 1: Immunohistochemistry of FLNC in ESCC tissues. (A) High FLNC expression was detected in the cytoplasm ESCC marginal portion cells (within $2 \mathrm{~mm}$ of the invasive external edge of tumor). Scale bar indicates $2 \mathrm{~mm}(\times 80)$; (B) magnified view $(\times 400)$. Images were captured by an Aperio CS2 scanner. (C) Low expression of FLNC $(\times 80)$; (D) magnified view $(\times 400)$.

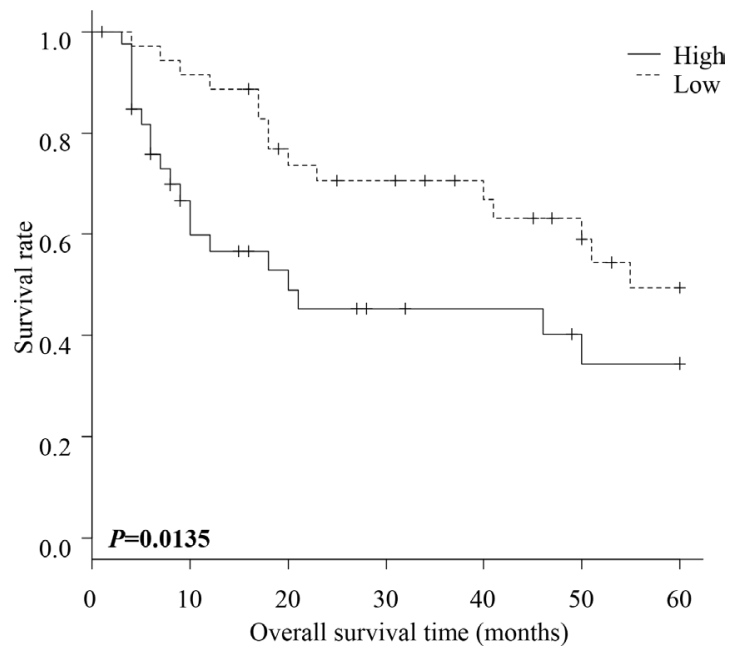

Figure 2: Overall survival of patients with ESCC. Overall survival of ESCC patients within high and low FLNC expression groups. The patients in the high FLNC expression group had significantly poorer prognoses than those in the low FLNC expression group $(P=0.0135)$. Survival rates were calculated using the Kaplan Meier method and differences in survival were estimated by Wilcoxon test. 
Table 2: Univariate and multivariate analysis in patients with ESCC for overall survival (Cox regression analysis)

\begin{tabular}{lcccc}
\hline & Univariate analysis & \multicolumn{3}{c}{ Multivariate analysis } \\
\cline { 2 - 5 } & $\boldsymbol{P}$-value & HR & $\mathbf{9 5 \%}$ CI & $\boldsymbol{P}$-value \\
\hline Gender & 0.366 & - & - & - \\
Histrogical grade (well/ mode, poor) & 0.485 & 0.803 & $0.39-1.65$ & 0.551 \\
pT & 0.202 & 2.508 & $0.86-7.34$ & 0.093 \\
pN & 0.063 & - & - & - \\
Lymphatic invasion & 0.009 & 2.942 & $1.20-7.19$ & 0.018 \\
Venous invasion & 0.218 & 0.618 & $0.25-1.52$ & 0.293 \\
pStage & 0.002 & - & - & - \\
FLNC expression (High/ Low) & 0.047 & 0.497 & $0.22-1.11$ & 0.088 \\
\hline
\end{tabular}

HR: hazard ratio, CI: confidence interval.

Univariate analyses and multivariate analysis were performed using Cox regression analysis. $P<0.05$ was considered statistically significant.

A
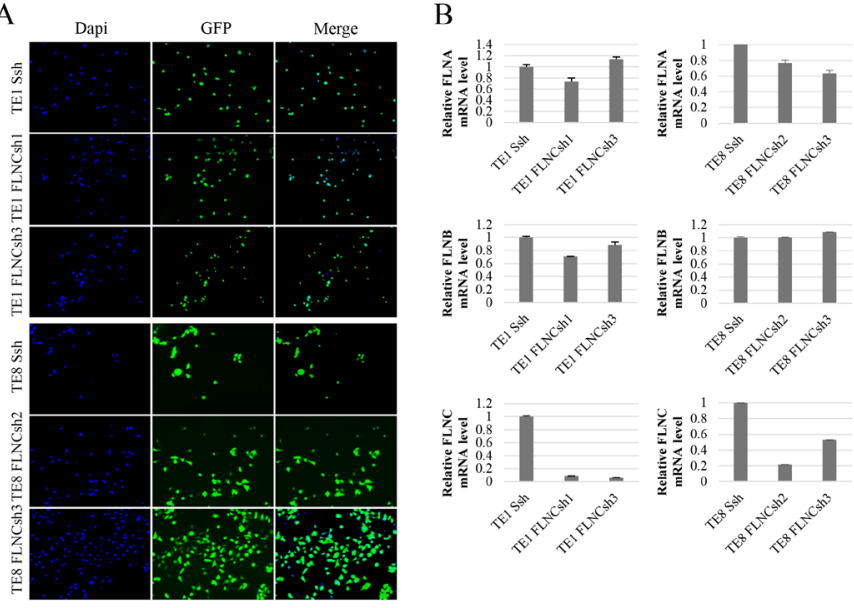

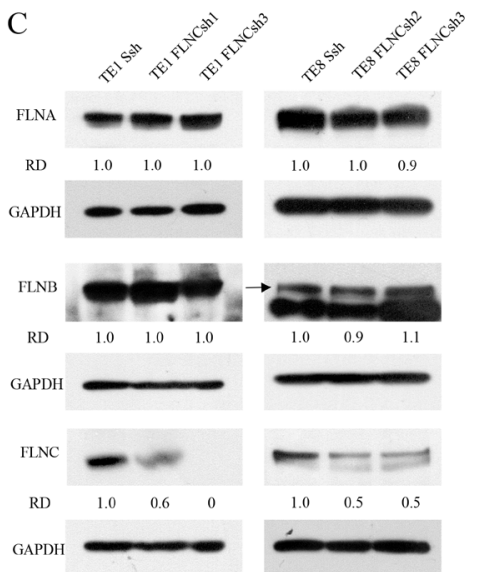

Figure 3: shRNA of FLNC specifically inhibits FLNC expression in shRNA infected ESCC cell lines. (A) Infection efficiency of shRNA lentiviral vectors in the ESCC cell lines (TE-1 and TE-8). Infection efficiency of shRNA lentiviral vectors was confirmed by visualization of green fluorescent protein (GFP) with a confocal microscope. Nuclei are stained with DAPI to visualize the cells. More than $90 \%$ of cells were confirmed to be infected with shRNA lentivirus. (B, C) mRNA and protein expression levels of FLNA, FLNB, and FLNC were examined by real-time PCR and immunoblotting. GAPDH was used as a loading control. The FLNA and FLNB expression were not reduced in FLNC shRNA infected cells, and were nearly identical to those of SshRNA infected cells. FLNC expression was clearly inhibited in FLNC shRNA infected cells as compared with its expression in SshRNA infected cells. Densities of the immunoblot bands were quantified using Image $\mathbf{J}$ software and normalized to GAPDH to obtain the relative densities (RD). 
was too low to detect in both TE- 1 and TE- 8 cells in our assays. Therefore, it is possible that Rac1 and $\mathrm{Cdc} 42$ activation are the driving forces of FLNC dependent ESCC cell motility. Several studies indicate that Rho GTPase is associated with lymphatic metastasis [36-38]. These reports support the correlation between our clinical and in vitro data. Therefore, FLNC potentially promotes lymphatic invasion and lymphatic metastasis by regulating Rac1 and Cdc42 activity in ESCC.
Matrix metalloproteinases (MMPs) are a family of structurally related zinc-dependent endopeptidases that are often increased in the tumor microenvironment and able to degrade various components of the ECM and lead to cancer cell invasion and metastasis [39-42]. MMPs also enhance the progression of the epithelialto-mesenchymal transition (EMT) [43, 44]. Therefore, MMPs are often associated with a poor prognosis of cancer patients $[39,45,46]$. MMPs are associated with
A

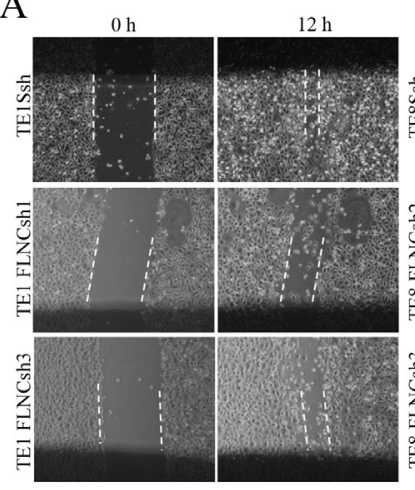

C
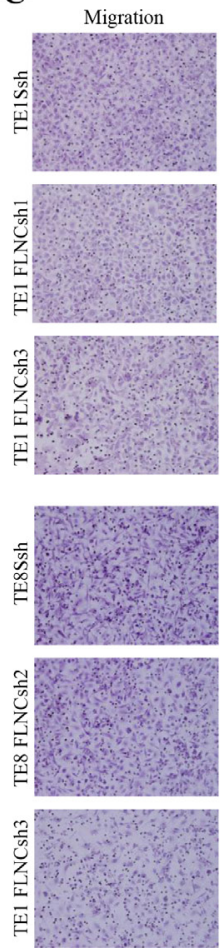

Invasion
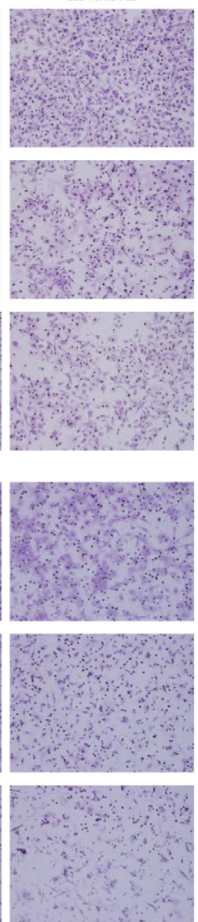

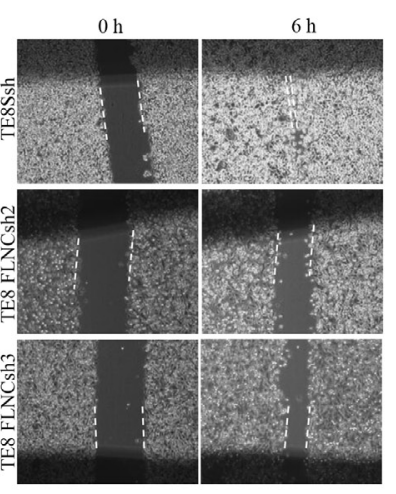

$\mathrm{D}$
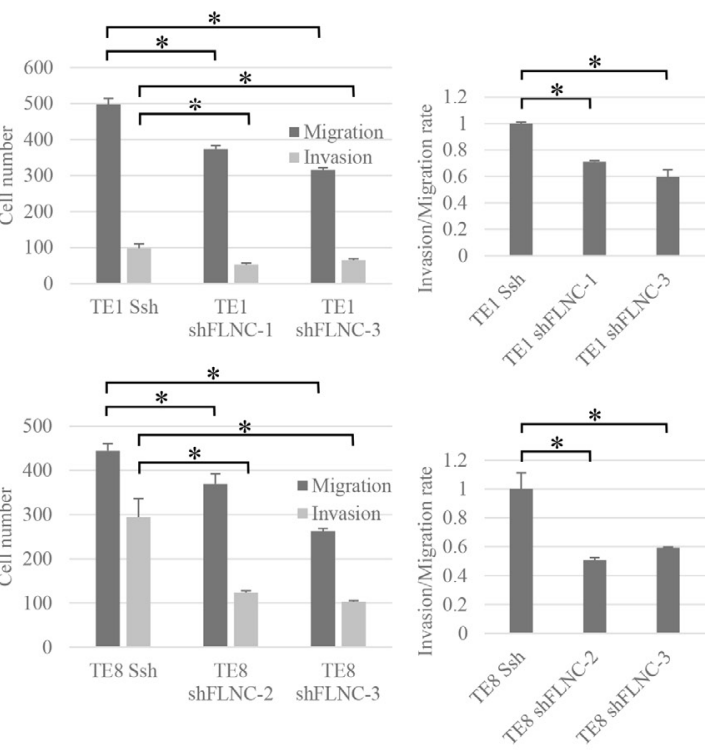

B
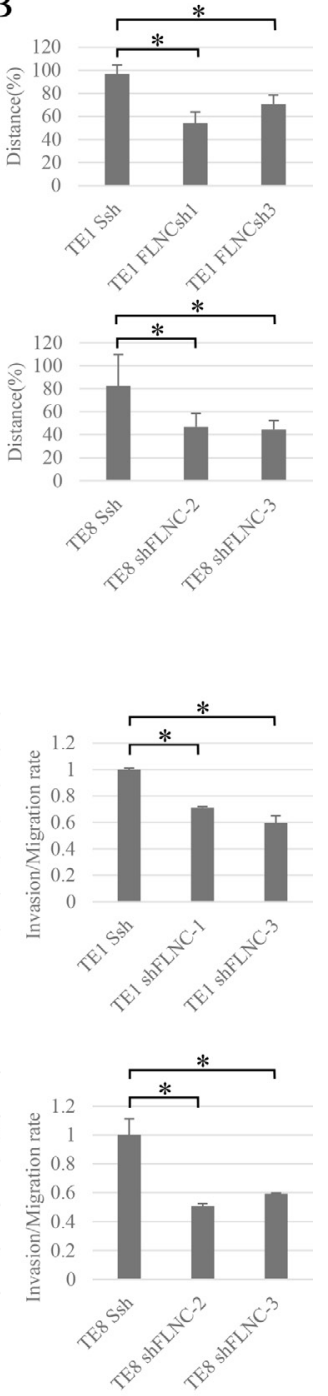

Figure 4: FLNC knockdown reduces cell migration and invasion activity. (A) Effect of FLNC knockdown in wound healing assay $(\times 40)$. The pictures show cells at 12 hours in TE-1 linage and 6 hours in TE- 8 linage after scraping. The dashed lines indicate the border of the cell free area. (B) The bar graphs indicate the widths of the cell free area of each cell type. FLNC knockdown inhibited cell migration significantly in both TE-1 and TE-8 cells. Columns represent three independent replicates and bars indicate SD. $* P<0.01$, significantly different from SshRNA infected cells. (C) The transwell migration and invasion assay $(\times 200)$ was used to examine the effect of FLNC knockdown on cell migration and invasion activity. (D) Quantification of migration and invasion abilities of FLNC knockdown cells. The numbers of migratory and invasive cells in the FLNC knockdown cells were significantly less than in control cells. The invasion/ migration rate of FLNC knockdown cells was also significantly less than the control cells. Columns represent total cell number in five independent microscopic fields and bars indicate SD. ${ }^{*} P<0.01$, significantly different from the number of SshRNA infected cells. 
clinicopathologic factors and prognosis in ESCC [47-49]. In addition, MMPs are also reported to be associated with Rho GTPase in cell invasion [50-52]. MMPs are expected to be associated with FLNC-Rac1 and cde42 axis and promote the invasion of ESCC in this study.

Our studies show that high FLNC expression in ESCC patients is correlated with lymphatic invasion, lymphatic metastasis, and an unfavorable prognosis. Additionally, we demonstrate that FLNC knockdown inhibited ESCC cell migration and invasion, possibly by regulating Rho GTPase. Our results suggest that FLNC might be a useful biomarker, and a promising therapeutic target, of lymphatic metastasis in ESCC patients. Future studies are required to define the mechanisms by which FLNC activates cell migration and invasion in ESCC.

\section{MATERIALS AND METHODS}

\section{Drugs, reagents, and antibodies}

The following reagents were purchased from the indicated manufacturers:

RPMI 1640 (Nikken Biomedical Laboratory, Osaka, Japan); fetal calf serum (FCS) (PAA Laboratories, Pasching, Austria); MTT (3-(4,5-dimethylthiazol-2-yl)-2,5-diphenyl tetrazolium bromide) (Sigma-Aldrich, St. Louis, MO, USA); DAPI (4',6-Diamidino-2-phenylindole, dihydrochloride, solution) (Dojindo Laboratories, Kumamoto, Japan); monoclonal antibodies against FLNA, GAPDH (EMD Millipore, Billerica, MA, USA; Cell Signaling Technology, Danvers, MA, USA, respectively), polyclonal antibodies against FLNB, FLNC (EMD Millipore, Billerica, MA, USA; Atlas Antibodies, Stockholm, Sweden, respectively).

\section{Patients and tumor samples}

The study included 75 consecutive patients with advanced ESCC (T2-4) who underwent surgical treatment in the Department of Digestive Surgery and Breast and Thyroid Surgery of Kagoshima University Hospital between January 2005 and January 2009. The clinical samples were obtained from tumors that were surgically removed and pathologically confirmed ESCC. The patients included 61 men and 14 women who ranged in age from 38 to 79 years old. None of the patients received preoperative radiotherapy or chemotherapy. The pathological features of ESCC were defined according to the TNM classification [3]. The study was approved by the Institutional Review Board of Kagoshima University, and performed in accordance with the Helsinki Declaration. Informed consent was obtained for each patient.

\section{Immunohistochemical analysis of patient tumors}

Surgical samples were fixed in $10 \%$ formaldehyde and embedded in paraffin before being cut into $3-\mu \mathrm{m}$ slices. Deparaffinization, hydrophilization, and target retrieval were performed in the PT Link system (Dako, Denmark). Endogenous peroxidase activity was blocked with 3\% hydrogen peroxide in methanol. After washing three times with PBS, the sections were preincubated in $1 \%$ bovine serum albumin for 30 minutes to block nonspecific reactions. The sections were incubated with FLNC rabbit polyclonal antibody (1:100 dilution) as the primary antibody overnight. Staining was performed using the avidin-biotin complex and immunoperoxidase method (Vectastatin ABC Kit, Vector Laboratories, Inc.,

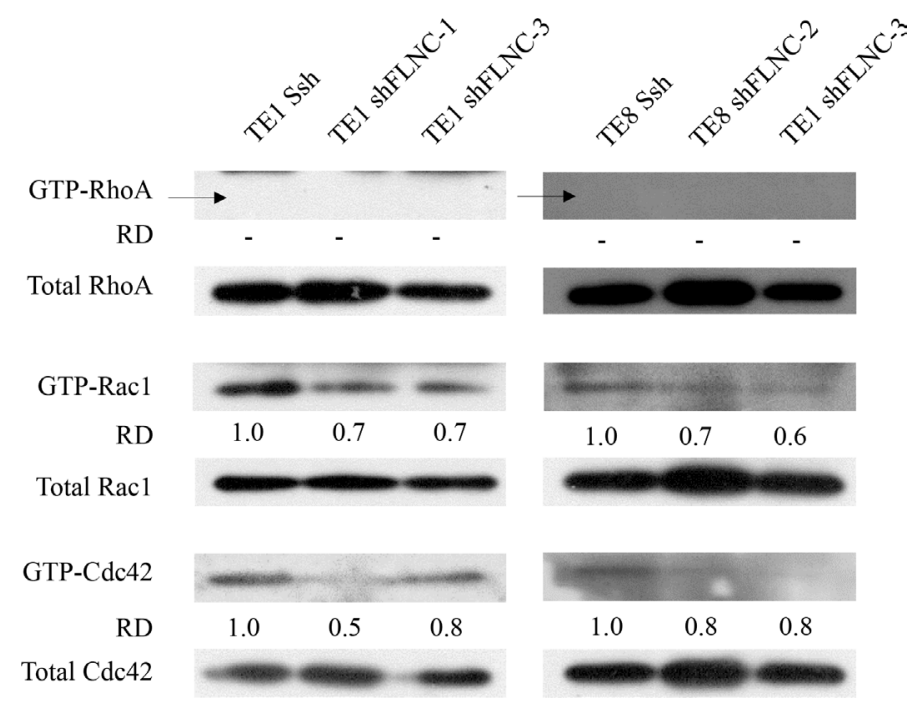

Figure 5: FLNC knock-down downregulates Rac1 and Cdc42 activation. Rho GTPase activities were evaluated in total lysates by activated GTPase pull-down. FLNC knockdown was associated with suppression of GTP-Rac1 and GTP-Cdc42, but not GTP-RhoA in both TE-1 and TE- 8 cells. Densities of the individual bands were quantified using Image J software. The densities of GTP-forms bands were normalized to the densities of bands of each total Rho family protein. Relative densities (RD)were obtained by comparing to the density of each GTP-form band of Ssh infected cells. 
Burlingame, CA, USA). The sections were visualized using diaminobenzidine tetraydrochloride. Images were captured using an Aperio CS2 scanner (Leica Biosystems, Nussloch, Germany). We evaluated FLNC expression in the marginal portions of the tumors, defined as within $2 \mathrm{~mm}$ in diameter of the invasive external edge of the tumor [26]. The number of cells in five microscopic fields (magnification $\times 200$ ) was counted independently by two researchers (K.T and Y.K). Positive cells were counted and ratios were obtained by dividing the number of immunopositive cells by the total number of cancer cells per field, and are expressed as a percentage.

\section{Cells and cell culture}

The human ESCC cell lines TE-1 and TE-8 were obtained from RIKEN BioResource Center (Tsukuba, Japan). Cells were cultured in RPMI 1640 supplemented with antibiotics (100 U / mL penicillin) and 10\% FCS. All cancer cell lines were cultured at $37^{\circ} \mathrm{C}$ in a $5 \% \mathrm{CO}_{2}$ humidified atmosphere.

\section{FLNC mRNA interference}

pENTR4-H1, CS-RfA-CG was obtained from RIKEN BioResource Center, Dr. Hiroyuki Miyoshi (Tsukuba, Japan). Oligonucleotides for FLNC protein suppression by shRNA (shRNA1, shRNA2, and shRNA3) interference were designed using siDirect (http://sidirect2. rnai.jp/) and obtained from FASMAC (Kanagawa, Japan). Sequences are provided in Supplementary Table S1. These oligonucleotides and Scrambled shRNA (SshRNA) [53] were annealed and ligated into the pENTR4-H1 entry vector using BglII and XbaI enzyme sites, respectively. The resulting pENTR4-H1-FLNC-shRNA1, pENTR4H1-FLNC-shRNA2, pENTR4-H1-FLNC-shRNA3, or pENTR4-H1-SshRNA vectors and the CS-RfA-CG destination vector were used in a recombination reaction using LR Clonase II (Invitrogen, Carlsbad, CA, USA) according to the manufacturer's instructions. 293FT cells (Invitrogen, Carlsbad, CA, USA) were plated in 6-well plates and transfected with the lentiviral vector plasmid (CS-FLNC-shRNA1-CG, CS-FLNC-shRNA2CG, CS-FLNC-shRNA3 or CS-SshRNA-CG) and lentivirus packaging plasmids (pMDLg/pRRE, pRSVRev, pMD2.G (Addgene, Cambridge, MA, USA)) using Lipofectamine 2000 (Invitrogen, Carlsbad, CA, USA) at $37^{\circ} \mathrm{C}$ for 48 hours. The supernatants, containing lentivirus, were concentrated to 10 times using Lenti-X ${ }^{\mathrm{TM}}$ Maxi Purification Kit (Clontech, Shiga, Japan). The ESCC cell lines plated in 6-well plates were infected by lentivirus at $37^{\circ} \mathrm{C}$ for 48 hours. After infection, cells were inoculated in a $10-\mathrm{cm}^{2}$ dish (500 cells / dish) for cloning. After seven days, colonies clearly derived from a single cell were selected based on GFP fluorescence.

\section{Cell proliferation assay}

Equal numbers of cells $\left(1 \times 10^{3}\right)$ were inoculated into each well and incubated for 1, 3, 5, and 7 days. Cell viability was measured using the MTT colorimetric assay [54].

\section{Wound healing assay}

A total of $4 \times 10^{5}$ cells were seeded in 12 well plates. After overnight incubation, cells were scraped with a $200-\mu \mathrm{L}$ pipette tip to make a straight-line cell-free scratch. Each well was washed with PBS to remove the remaining unattached cells. The cells were observed and pictures were taken 12 hours (TE-1 linage) and 6 hours (in TE-8 linage) after scraping. Cell motility was quantified by measuring the distance between the migrating cell boundaries.

\section{Transwell migration and invasion assay}

Migration and invasion assays were performed using transwell chambers. For invasion assays, we used BioCoat $^{\mathrm{TM}}$ Matrigel Invasion Chamber 24-well $8 \mu \mathrm{m}$ (Corning, NY, USA). Cells $\left(1 \times 10^{5}\right)$ were seeded with serum free medium onto the top chamber, and the bottom chamber was filled with medium including $10 \%$ FCS and $1 \mathrm{ng} / \mathrm{mL}$ EGF (PeproTech, Rocky Hill, NJ, USA). For the migration assays, we used BioCoat ${ }^{\mathrm{TM}}$ Control Inserts 24 well $8 \mu \mathrm{m}$. A chamber membrane was coated with $10 \mu \mathrm{g} / \mathrm{mL}$ fibronectin (Sigma-Aldrich, St. Louis, MO, USA). After 48 hours of seeding the cells, the bottom membranes were fixed using 4\% paraformaldehyde, and stained with hematoxylin. Cell numbers were quantified by counting 5 random fields under a microscope at a magnification of $200 \times$.

\section{RNA isolation and cDNA synthesis}

Total RNA from the cultured cells was isolated using TRIzol (Invitrogen, Carlsbad, CA, USA), and reversetranscribed using the ReverTra Ace kit (Toyobo, Osaka, Japan), according to the manufacturer's instructions [55].

\section{Quantitative real-time PCR}

mRNA expression levels of FLNA, FLNB, and FLNC were determined by real-time RT-PCR (Step One Plus ${ }^{\mathrm{TM}}$; Applied Biosystems, Foster City, CA, USA) using Go Taq qPCR Master Mix (Promega, Wisconsin, USA) according to the manufacturer's instructions. Human GAPDH was used for normalization. The expression of the target gene was quantified using the comparative cycle threshold method. The respective forward and reverse primer sequences are provided in Supplementary Table S2. 


\section{Protein extraction and immunoblotting}

Total cell lysate was isolated with RIPA buffer (25 mM Tris-HCl (pH 7.5), $150 \mathrm{mM} \mathrm{NaCl}, 1 \%$ Nonidet P-40 (NP-40), 0.1\% SDS, $0.5 \%$ sodium deoxycholate, $1 \mathrm{mM}$ p-amidinophenyl methanesulfonyl fluoride hydrochloride (APMSF), and $1 \mu \mathrm{g} / \mathrm{mL}$ aprotinin). Protein concentrations were measured using the BioRad protein assay kit (Hercules, CA, USA). Cell lysates $(20 \mu \mathrm{g}$ protein for FLNA, and $50 \mu \mathrm{g}$ protein for FLNB and FLNC) were separated by $5 \%-20 \%$ SDS-polyacrylamide gel (ATTO, Tokyo, Japan) electrophoresis and transferred onto membranes as described previously [56]. The blotted membranes were incubated with anti-FLNA (1:500000 dilution), anti-FLNB (1:1000 dilution), antiFLNC (1:500 dilution), and GAPDH (1:100000 dilution) antibody overnight at $4{ }^{\circ} \mathrm{C}$, and each protein was detected as described previously [56]. Protein expression was quantified using Image $J$ software (National Institutes of Mental Health, Bethesda, MD, USA) and normalized to GAPDH to obtain the relative densities (RD).

\section{Small GTPase pull-down assay}

Small GTPase pull-down assay was performed using the RhoA/Rac1/Cdc42 Activation Assay Combo Kit (Cell BioLabs, San Diego, CA, USA) following the manufacturer's instructions. Cells were incubated with medium and $1 \mathrm{ng} / \mathrm{mL}$ EGF for 2-4 days until they obtained approximately $80-90 \%$ confluence. Cell lysates were incubated with Rhotekin RBD (for RhoA) or PAK1 PBD (for Rac1/Cdc42) agarose beads for 1 hour at $4^{\circ} \mathrm{C}$. GTP-RhoA, GTP-Rac1, or GTP-Cde42 was detected by immunoblotting.

\section{Statistical analysis}

All of the statistical calculations were carried out with EZR (http://www.jichi.ac.jp/saitama-sct/) [57]. Statistical analyses of group differences were performed using the $\chi^{2}$-test and the Student's $t$-test. Patients were divided into FLNC expression high and low groups based on median FLNC expression levels. Kaplan-Meier survival curves were generated comparing these two groups via the Wilcoxson test. Univariate analysis and multivariate analysis were evaluated by Cox regression analysis. Differences were considered significant at $P<0.05$.

\section{ACKNOWLEDGMENTS}

The authors thank: Dr. Hiroyuki Miyoshi (Keio University) and Riken BioResource Center for lentivirus vectors; Ms. Hiromi Mitsuo and Ms. Naoko Hidaka for their excellent secretarial assistance; and Ms. Aya Harada and Ms. Mika Tokunaga for their excellent technical assistance. We also wish to thank the Joint Research
Laboratory, Kagoshima University Graduate School of Medical and Dental Sciences, for the use of their facilities. This work was supported by JSPS KAKENHI Grant Number JP26870456, JP15K18413, JP16K07121, JP26670160, JP25430114, Mitsui Life Social Welfare Foundation and a grant from Kodama Memorial Fund for Medical Research. And, This Work was supported in part by a grant from the Graduate School of Medical and Dental Sciences, Kagoshima University.

\section{CONFLICTS OF INTEREST}

The authors have no conflicts of interest to disclose.

\section{FUNDING}

This work was supported by JSPS KAKENHI Grant Number JP26870456, JP15K18413, JP16K07121, JP26670160, JP25430114, Mitsui Life Social Welfare Foundation and a grant from Kodama Memorial Fund for Medical Research.

\section{REFERENCES}

1. Aznavoorian S, Murphy AN, Stetler-Stevenson WG, Liotta LA. Molecular aspects of tumor cell invasion and metastasis. Cancer. 1993; 71:1368-1383.

2. Fidler IJ. Tumor heterogeneity and the biology of cancer invasion and metastasis. Cancer Res. 1978; 38:2651-2660.

3. Sobin LH, Fleming ID. TNM classification of malignant tumors, fifth edition (1997). Cancer. 1997; 80:1803-1804.

4. Pollard TD, Borisy GG. Cellular motility driven by assembly and disassembly of actin filaments. Cell. 2003; 112:453-465.

5. Wang K, Ash JF, Singer SJ. Filamin, a new high-molecularweight protein found in smooth muscle and non-muscle cells. Proc Natl Acad Sci USA. 1975; 72:4483-4486.

6. Baldassarre M, Razinia Z, Burande CF, Lamsoul I, Lutz PG, Calderwood DA. Filamins regulate cell spreading and initiation of cell migration. PLoS One. 2009; 4:e7830.

7. van der Flier A, Sonnenberg A. Structural and functional aspects of filamins. Biochim Biophys Acta. 2001; 1538:99-117.

8. Feng Y, Walsh CA. The many faces of filamin: a versatile molecular scaffold for cell motility and signalling. Nat Cell Biol. 2004; 6:1034-1038.

9. Popowicz GM, Schleicher M, Noegel AA, Holak TA. Filamins: promiscuous organizers of the cytoskeleton. Trends Biochem Sci. 2006; 31:411-419.

10. Stossel TP, Hartwig JH. Filling gaps in signaling to actin cytoskeletal remodeling. Dev Cell. 2003; 4:444-445.

11. Zhou AX, Toylu A, Nallapalli RK, Nilsson G, Atabey N, Heldin CH, Boren J, Bergo MO, Akyurek LM. Filamin a mediates $\mathrm{HGF} / \mathrm{c}-\mathrm{MET}$ signaling in tumor cell migration. Int J Cancer. 2011; 128:839-846. 
12. Cunningham CC, Gorlin JB, Kwiatkowski DJ, Hartwig JH, Janmey PA, Byers HR, Stossel TP. Actin-binding protein requirement for cortical stability and efficient locomotion. Science. 1992; 255:325-327.

13. van der Flier A, Kuikman I, Kramer D, Geerts D, Kreft M, Takafuta T, Shapiro SS, Sonnenberg A. Different splice variants of filamin-B affect myogenesis, subcellular distribution, and determine binding to integrin [beta] subunits. J Cell Biol. 2002; 156:361-376.

14. Ai J, Huang H, Lv X, Tang Z, Chen M, Chen T, Duan W, Sun H, Li Q, Tan R, Liu Y, Duan J, Yang Y, et al. FLNA, PGK1 are two potential markers for progression in hepatocellular carcinoma. Cell Physiol Biochem. 2011; 27:207-216.

15. Sun GG, Lu YF, Cheng YJ, Hu WN. Absent expression of FLNA is correlated with poor prognosis of nasopharyngeal cancer. Tumour Biol. 2014; 35:2967-2974.

16. Sun GG, Sheng SH, Jing SW, Hu WN. An antiproliferative gene FLNA regulates migration and invasion of gastric carcinoma cell in vitro and its clinical significance. Tumour Biol. 2014; 35:2641-2648.

17. Bandaru S, Zhou AX, Rouhi P, Zhang Y, Bergo MO, Cao Y, Akyurek LM. Targeting filamin B induces tumor growth and metastasis via enhanced activity of matrix metalloproteinase-9 and secretion of VEGF-A. Oncogenesis. 2014; 3:e119.

18. Shi J, Zhang G, Yao D, Liu W, Wang N, Ji M, He N, Shi B, Hou P. Prognostic significance of aberrant gene methylation in gastric cancer. Am J Cancer Res. 2012; 2:116-129.

19. Adachi-Hayama M, Adachi A, Shinozaki N, Matsutani T, Hiwasa T, Takiguchi M, Saeki N, Iwadate Y. Circulating anti-filamin $\mathrm{C}$ autoantibody as a potential serum biomarker for low-grade gliomas. BMC Cancer. 2014; 14:452.

20. Amaro A, Esposito AI, Gallina A, Nees M, Angelini G, Albini A, Pfeffer U. Validation of proposed prostate cancer biomarkers with gene expression data: a long road to travel. Cancer Metastasis Rev. 2014; 33:657-671.

21. Qiao J, Cui SJ, Xu LL, Chen SJ, Yao J, Jiang YH, Peng G, Fang CY, Yang PY, Liu F. Filamin C, a dysregulated protein in cancer revealed by label-free quantitative proteomic analyses of human gastric cancer cells. Oncotarget. 2015; 6:1171-1189. doi: 10.18632/oncotarget.2645.

22. Ferlay J, Soerjomataram I, Dikshit R, Eser S, Mathers C, Rebelo M, Parkin DM, Forman D, Bray F. Cancer incidence and mortality worldwide: sources, methods and major patterns in GLOBOCAN 2012. Int J Cancer. 2015; 136:E359-386.

23. Baba M, Aikou T, Yoshinaka H, Natsugoe S, Fukumoto T, Shimazu H, Akazawa K. Long-term results of subtotal esophagectomy with three-field lymphadenectomy for carcinoma of the thoracic esophagus. Ann Surg. 1994; 219:310.

24. Enzinger PC, Mayer RJ. Esophageal cancer. N Engl J Med. 2003; 349:2241-2252.
26. Hase K, Shatney C, Johnson D, Trollope M, Vierra M. Prognostic Value of Tumor Budding in Patients with Colorectal-Cancer. Dis Colon Rectum. 1993; 36:627-635.

26. Kurahara H, Takao S, Maemura K, Shinchi H, Natsugoe S, Aikou T. Impact of Vascular Endothelial Growth Factor-C and -D Expression in Human Pancreatic Cancer: Its Relationship to Lymph Node Metastasis. Clin Cancer Res. 2004; 10:8413-8420.

27. Kamochi N, Nakashima M, Aoki S, Uchihashi K, Sugihara H, Toda S, Kudo S. Irradiated fibroblast-induced bystander effects on invasive growth of squamous cell carcinoma under cancer-stromal cell interaction. Cancer Sci. 2008; 99:2417-2427.

28. Marti A, Luo Z, Cunningham C, Ohta Y, Hartwig J, Stossel TP, Kyriakis JM, Avruch J. Actin-binding protein-280 binds the stress-activated protein kinase (SAPK) activator SEK-1 and is required for tumor necrosis factor-alpha activation of SAPK in melanoma cells. J Biol Chem. 1997; 272:2620-2628.

29. Zhou AX, Hartwig JH, Akyurek LM. Filamins in cell signaling, transcription and organ development. Trends Cell Biol. 2010; 20:113-123.

30. Hall A. Rho GTPases and the actin cytoskeleton. Science. 1998; 279:509-514.

31. Bishop AL, Hall A. Rho GTPases and their effector proteins. Biochem J. 2000; 348:241-255.

32. Aspenstrom P, Fransson A, Saras J. Rho GTPases have diverse effects on the organization of the actin filament system. Biochem J. 2004; 377:327-337.

33. Machacek M, Hodgson L, Welch C, Elliott H, Pertz O, Nalbant P, Abell A, Johnson GL, Hahn KM, Danuser G. Coordination of Rho GTPase activities during cell protrusion. Nature. 2009; 461:99-103.

34. Le Clainche C, Carlier MF. Regulation of actin assembly associated with protrusion and adhesion in cell migration. Physiol Rev. 2008; 88:489-513.

35. Mattila PK, Lappalainen P. Filopodia: molecular architecture and cellular functions. Nat Rev Mol Cell Biol. 2008; 9:446-454.

36. Feng JG, Liu Q, Qin X, Geng YH, Zheng ST, Liu T, Sheyhidin I, Lu XM. Clinicopathological pattern and Annexin A 2 and $\mathrm{Cdc} 42$ status in patients presenting with differentiation and lymphnode metastasis of esophageal squamous cell carcinomas. Mol Biol Rep. 2012; 39:1267-1274.

37. Shida A, Fujioka S, Takahashi N, Aoki H, Okamoto T, Mitsumori N, Omura N, Yanaga K. Reduced expression of Rho GDP dissociation inhibitor 2 mRNA is associated with lymph node metastasis in gastric carcinoma. Oncol Lett. 2013; 6:463-467.

38. Yang Q, Luo GY, Li Y, Shan HB, Wang HY, Xu GL. Expression of Rac-1 related to tumor depth, lymph node metastasis and patient prognosis in esophageal squamous cell carcinoma. Med Oncol. 2013; 30:689. 
39. Vihinen P, Kahari VM. Matrix metalloproteinases in cancer: prognostic markers and therapeutic targets. Int J Cancer. 2002; 99:157-166.

40. Maskos K, Bode W. Structural basis of matrix metalloproteinases and tissue inhibitors of metalloproteinases. Mol Biotechnol. 2003; 25:241-266.

41. Nagase H, Visse R, Murphy G. Structure and function of matrix metalloproteinases and TIMPs. Cardiovasc Res. 2006; 69:562-573.

42. Page-McCaw A, Ewald AJ, Werb Z. Matrix metalloproteinases and the regulation of tissue remodelling. Nat Rev Mol Cell Biol. 2007; 8:221-233.

43. Radisky ES, Radisky DC. Matrix metalloproteinaseinduced epithelial-mesenchymal transition in breast cancer. J Mammary Gland Biol Neoplasia. 2010; 15:201-212.

44. van Zijl F, Krupitza G, Mikulits W. Initial steps of metastasis: cell invasion and endothelial transmigration. Mutat Res. 2011; 728:23-34.

45. Curran S, Dundas SR, Buxton J, Leeman MF, Ramsay R, Murray GI. Matrix metalloproteinase/tissue inhibitors of matrix metalloproteinase phenotype identifies poor prognosis colorectal cancers. Clin Cancer Res. 2004; 10:8229-8234.

46. Gu ZD, Li JY, Li M, Gu J, Shi XT, Ke Y, Chen KN. Matrix metalloproteinases expression correlates with survival in patients with esophageal squamous cell carcinoma. Am J Gastroenterol. 2005; 100:1835-1843.

47. Shima I, Sasaguri Y, Kusukawa J, Yamana H, Fujita H, Kakegawa T, Morimatsu M. Production of matrix metalloproteinase-2 and metalloproteinase-3 related to malignant behavior of esophageal carcinoma. A clinicopathologic study. Cancer. 1992; 70:2747-2753.

48. Koyama H, Iwata H, Kuwabara Y, Iwase H, Kobayashi S, Fujii Y. Gelatinolytic activity of matrix metalloproteinase-2 and-9 in oesophageal carcinoma; a study using in situ zymography. Eur J Cancer. 2000; 36:2164-2170.

49. Li Y, Ma J, Guo Q, Duan F, Tang F, Zheng P, Zhao Z, Lu G. Overexpression of MMP-2 and MMP-9 in esophageal squamous cell carcinoma. Dis Esophagus. 2009; 22:664-667.
50. Kheradmand F, Werner E, Tremble P, Symons M, Werb Z. Role of Rac1 and oxygen radicals in collagenase-1 expression induced by cell shape change. Science. 1998; 280:898-902.

51. Zhuge Y, Xu J. Rac1 mediates type I collagen-dependent MMP-2 activation. role in cell invasion across collagen barrier. J Biol Chem. 2001; 276:16248-16256.

52. Deroanne CF, Hamelryckx D, Ho TT, Lambert CA, Catroux P, Lapiere CM, Nusgens BV. Cdc42 downregulates MMP-1 expression by inhibiting the ERK1/2 pathway. J Cell Sci. 2005; 118:1173-1183.

53. Sasaki M, Kawahara K, Nishio M, Mimori K, Kogo R, Hamada K, Itoh B, Wang J, Komatsu Y, Yang YR, Hikasa H, Horie Y, Yamashita T, et al. Regulation of the MDM2-P53 pathway and tumor growth by PICT1 via nucleolar RPL11. Nat Med. 2011; 17:944-951.

54. Mosmann T. Rapid Colorimetric Assay for Cellular Growth and Survival - Application to Proliferation and CytoToxicity Assays. J Immunol Methods. 1983; 65:55-63.

55. Shinsato Y, Furukawa T, Yunoue S, Yonezawa H, Minami K, Nishizawa Y, Ikeda R, Kawahara K, Yamamoto M, Hirano H, Tokimura H, Arita K. Reduction of MLH1 and PMS2 confers temozolomide resistance and is associated with recurrence of glioblastoma. Oncotarget. 2013; 4:2261-2270. doi: 10.18632/oncotarget.1302.

56. Che XF, Zheng CL, Owatari S, Mutoh M, Gotanda T, Jeung HC, Furukawa T, Ikeda R, Yamamoto M, Haraguchi M, Arima N, Akiyama S. Overexpression of survivin in primary ATL cells and sodium arsenite induces apoptosis by down-regulating survivin expression in ATL cell lines. Blood. 2006; 107:4880-4887.

57. Kanda Y. Investigation of the freely available easy-to-use software 'EZR' for medical statistics. Bone Marrow Transplant. 2013; 48:452-458. 